\title{
Auditory temporal perception deficits in the reading-impaired: A critical review of the evidence
}

\author{
MICHAEL STUDDERT-KENNEDY \\ Haskins Laboratories, New Haven, Connecticut \\ and \\ MARIA MODY \\ Albert Einstein College of Medicine, New York, New York
}

\begin{abstract}
We assess evidence and arguments brought forward by Tallal (e.g., 1980) and by the target paper (Farmer \& Klein, 1995) for a general deficit in auditory temporal perception as the source of phonological deficits in impaired readers. We argue that (1) errors in temporal order judgment of both syllables and tones reflect difficulty in identifying similar (and so readily confusable) stimuli rapidly, not in judging their temporal order; (2) difficulty in identifying similar syllables or tones rapidly stem from independent deficits in speech and nonspeech discriminative capacity, not from a general deficit in rate of auditory perception; and (3) the results of dichotic experiments and studies of aphasics purporting to demonstrate left-hemisphere specialization for nonspeech auditory temporal perception are inconclusive. The paper supports its arguments with data from a recent control study. We conclude that, on the available evidence, the phonological deficit of impaired readers cannot be traced to any co-occurring nonspeech deficits so far observed and is phonetic in origin, but that its full nature, origin, and extent remain to be determined.
\end{abstract}

The target paper (Farmer \& Klein, 1995) starts from the widely accepted assumption (with which we agree) that dyslexia, or reading impairment, is often, if not always, associated with a phonological deficit. The stated purpose of the paper is, then, to "evaluate the plausibility" of the hypothesis of Tallal (1984) that this deficit is "a symptom of an underlying auditory temporal processing deficit" (abstract). Unfortunately, this hypothesis has never been clearly or consistently framed by Tallal herself, and Farmer and Klein do nothing to clarify it. Much of what we have to say therefore entails analysis of Tallal's work no less than that of Farmer and Klein. Our remarks are limited to studies of audition, because these alone bear on possible weaknesses in speech perception from which a phonological deficit might arise.

As best we can determine, Tallal's hypothesis, originally a proposal concerning the perceptual deficits of dysphasic children, has come to comprise three logically independent, but mutually reinforcing, propositions (for a recent review, see Tallal, Miller, \& Fitch, 1993): (1) "Rapid auditory temporal processing" is essential to speech perception; (2) specialization of the left cerebral hemisphere for speech perception (and so for phonology), in most righthanded individuals, is grounded in a prior specialization of that hemisphere for "rapid auditory temporal processing";

Preparation of this paper was supported in part by NICHD Grant HD01994 to Haskins Laboratories. We thank Susan Brady for useful discussion and James Neely for judicious editorial advice. Correspondence should be addressed to $\mathrm{M}$. Studdert-Kennedy, Haskins Laboratories, 270 Crown St., New Haven, CT 0651 1-6695 (e-mail: haskins@haskins. yale.edu).
(3) phonological deficits in some dysphasic children, some aphasic adults, and some impaired readers, or dyslexics, stem from deficits in "rapid auditory temporal processing." For Tallal and her colleagues, the first proposition, though far from clear, seems to be axiomatic. They have given most attention to the third proposition, rather less to the second. Farmer and Klein follow this distribution of emphasis, and we largely follow the target paper. But all three propositions seem to be required for a full statement of the hypothesis.

\section{BACKGROUND}

We begin by distinguishing two concepts repeatedly confused both in the work of Tallal (e.g., Tallal \& Newcombe, 1978; see below) and in the target paper (Farmer \& Klein, 1995): temporal perception (or "processing") and rapid perception. In normal linguistic usage, temporal perception contrasts with, say, spectral perception in audition or spatial perception in vision; it refers to perceiving the temporal properties of events (duration, sequence, relative timing, rhythm). To the extent that they identify temporal perception with sequential perception, Farmer and Klein (1995) follow this usage. However, when they equate temporal perception with "processing rapidly presented stimuli" (p. 476; see also p. 460) or with perceiving "spectral changes in the time frame of tens of milliseconds" (p. 467), they confuse rate of perception with perception of rate. Perception is "temporal" if the defining property of the perceived event is temporal; it does not become "temporal" by virtue of being effected rapidly. This distinction is not a trivial matter of terminology; it is conceptually and substantively 
important, because the precise nature of a speech perceptual deficit bears directly on what correlated deficits we might expect in speech production, on the nature of the underlying defective neural mechanism, and on how we might go about remediation. Let us see, then, how the confusion has arisen.

In a series of studies from which much of Tallal's later work springs (see the review by Tallal et al., 1993), Tallal and Piercy $(1973,1974,1975)$ compared dysphasic children with normal controls on tests of discrimination and temporal order judgment (TOJ) for pairs of stimuli presented with "long" (428 msec) and "short" (8-305 msec) interstimulus intervals (ISI). The stimuli included (1) short (75-msec) and long (250-msec) complex tones differing in fundamental frequency (100 vs. $305 \mathrm{~Hz})$; (2) long (250msec) steady-state synthetic vowels $(/ \varepsilon /, / æ /)$; (3) short (43-msec) steady-state, synthetic vowels $(/ \mathcal{E} /, / \mathfrak{x} /)$ immediately followed by a longer $(207-\mathrm{msec})$ steady-state synthetic vowel (/I/); and (4) synthetic consonant-vowel (CV) syllables ( $/ \mathrm{ba} / \mathrm{vs}$. $/ \mathrm{da} /, 250 \mathrm{msec})$, in which the contrasting second $(F 2)$ and third $(F 3)$ formant transitions and the noncontrasting first $(F 1)$ formant transitions, at syllable onset, were all either short (43 msec) or long (95 msec) in duration. The dysphasic children performed significantly worse than normals on short tones, short vowels, and short transition consonants at short ISIs but not on the corresponding long stimuli or the long ISIs. Moreover, performance on discrimination and TOJ did not differ significantly. From this last finding, Tallal and Piercy (1973, p. 396) inferred that apparent deficits in auditory sequencing could be due to difficulty in discriminating and identifying stimuli rapidly, rather than to deficits in temporal perception itself. From the similar results for short steadystate vowels and short transition consonants, and from improved performance on long transition consonants, ${ }^{1}$ they concluded that "it is the brevity not the transitional character... [of the formant transitions] of synthesized stop consonants which results in the impaired perception of our dysphasic children" (Tallal \& Piercy, 1975, p. 73; our italics). And, from the similar results for short tones and short speech sounds, they concluded that the dysphasics' impairment was a general auditory deficit, not specific to speech (Tallal \& Piercy, 1973, 1974). These three conclusions directly address three main issues in Farmer and Klein's paper concerning auditory temporal perception. Although Farmer and Klein cite Tallal and Piercy (1973, 1975, but not 1974), they do not comment on these conclusions. Nonetheless, let us examine each in turn.

Discrimination, not TOJ. The most important conclusion, in the present context, is that dysphasic children's difficulties were with discrimination, not with TOJ itself. Similarly, Tallal (1980), in her only study of specifically reading-impaired children, again found that discrimination and TOJ of complex tones did not differ significantly, and again concluded that the children's "difficulty with temporal pattern perception may stem from a more primary [sic] perceptual deficit that affects the rate at which they can process perceptual information" (Tallal, 1980, p. 193; our italics; see Tallal, Sainburg, \& Jernigan, 1991, p. 365, for a recent restatement of this view). Notice that on this account a slowed rate of perception, as indicated by errors on TOJ at short ISIs, is not a general cause of the impaired child's difficulties, but a specific result of low discriminative capacity along a particular dimension. Reed (1989), the only other researcher to extend Tallal's TOJ tests to reading-impaired children, concurs, proposing that TOJ not be viewed as a measure of temporal processing at all: "The temporal task simply provides a setting where perceptual capabilities can be stressed, allowing the measurement of differences in the absence of ceiling performance" (Reed, 1989, p. 287). In other words, the TOJ task is primarily a diagnostic tool for picking up subtle deficits in discriminative capacity, and these deficits reveal themselves in a slowed rate of perception, specific to the dimension being tested. If this view is correct, as we believe it is, we have no experimental evidence at all for deficits either in auditory temporal perception or in general rate of auditory perception among the reading-impaired. Again, although Farmer and Klein cite Tallal (1980) and Reed (1989), they do not report these conclusions or consider how their work tempers the argument of the target paper.

Perceiving formant transitions: The brevity of transitions, not their transitional character (i.e., not acoustic changes), causes the perceptual difficulty. This conclusion contradicts the claim that supposed deficits in perception of formant transitions stem from difficulty in perceiving "rapid acoustic changes." As noted in the next paragraph, no subsequent experimental work has established that claim.

The relation between deficits in speech and nonspeech auditory perception. Tallal and Piercy $(1973,1974)$ demonstrated that dysphasic children suffered from deficits in both speech (phonetic) and nonspeech (auditory) discrimination, but not that the latter caused the former, as an auditory account of phonological deficits would require. Later papers (Schwartz \& Tallal, 1980; Tallal \& Newcombe, 1978), which we analyze in some detail below, tried to close the gap by proposing that the phonological capacities of the left cerebral hemisphere rested on capacities for "auditory temporal processing," presumed deficient in certain clinical populations. Yet neither Tallal and Piercy (1974) nor anyone else has demonstrated that difficulties with $/ \mathrm{ba} /-/ \mathrm{da} /$ discrimination are auditory rather than phonetic, because no one has demonstrated equivalent difficulties for matched nonspeech control patterns with brief and rapidly changing onset frequencies. Similarly, we cannot attribute difficulties in discriminating $/ \mathrm{ba} /-/ \mathrm{da} /$ or $/ \varepsilon /-/ \mathfrak{a} /$ to concomitant difficulties in discriminating the fundamental frequency of complex nonspeech tones, because neither speech contrast depends on variations in fundamental frequency.

\section{A CONCEPTUAL MUDDLE}

In light of the foregoing, we may well be puzzled by Tallal's continued use of such phrases as "temporal processing disorder" (e.g., Tallal et al., 1991, p. 363) and deficits in "the processing of rapidly changing acoustic spec- 
tra" (e.g., Tallal et al., 1993, p. 40) to describe the condition of poor readers, and by Farmer and Klein's uncritical acceptance of this terminology. To understand how this usage has come about, we must turn to a paper, not mentioned by Farmer and Klein, that extended Tallal's TOJ tests to adults with brain lesions due to missile wounds (Tallal \& Newcombe, 1978). We discuss the findings of this paper below (see Aphasic studies). Here, our immediate interest is in how the authors describe the work of Tallal and Piercy $(1973,1974,1975)$. Two inconsistencies deserve note. First, despite the earlier conclusion that the dysphasic children's difficulties were with identifying stimuli at rapid rates of presentation rather than with temporal perception itself, the introduction to the new paper states that these earlier studies "strongly support the hypothesis that some developmental language disorders may result from a primary impairment in auditory temporal analysis" (Tallal \& Newcombe, 1978, p. 13). From this point on, the phrases "impaired on auditory temporal processing tasks" (p. 14), "defect in temporal acoustic processing" (p. 22), and the like, are used interchangeably with "impairment in responding to rapidly presented acoustic information" (p. 14). No justification is offered either for this conflation of perceiving the temporal properties of events with perceiving brief events rapidly or for the switch in interpretation from that of Tallal and Piercy (1973).

The second inconsistency is no less serious. Despite Tallal \& Piercy's (1975) conclusion that "it is the brevity not the transitional character" (p. 73) of formant transitions that causes difficulty for dysphasic children, Tallal and Newcombe (1978) attribute the children's difficulty to "speech sounds that incorporate rapidly changing acoustic spectra" (p. 13). Thus, without any new evidence, they adopt the very interpretation that Tallal and Piercy (1975) tested and rejected. Tallal and Newcombe (1978) do not acknowledge this reversal, and so can hardly justify, or even explain, it. We can infer the underlying rationale, however, from their equation of discrete pitch changes in the tone TOJ test with the continuously changing spectral sweeps of stop-vowel formant transitions. The equation itself, also not explicitly acknowledged, we infer from several passing remarks. First, the authors characterize the difficulties of Tallal and Piercy's (1973) dysphasic children with tone TOJ as "imperception of rapidly changing nonverbal acoustic material" (Tallal \& Newcombe, 1978, p. 13). Second, they state that their adult subjects with left-hemisphere damage were "impaired in their ability to respond correctly to rapidly changing acoustic stimuli, regardless of whether stimuli were verbal or nonverbal"' (p. 13). Yet the only verbal stimuli with which the patients had difficulty were stop consonant-vowel syllables, while the only nonverbal stimuli with which they were tested were the discrete tones of the tone TOJ test.

Finally, we infer the equation of tones and transitions from the claim, based on the performance of their aphasic subjects on the TOJ tests, that "the left hemisphere must play a primary role in the analysis of specific rapidly changing acoustic cues, verbal and nonverbal, and ... such analysis is critically involved in both the development and maintainance [sic] of language" (Tallal \& Newcombe, 1978, p. 19). Tallal and Newcombe support this claim by referring to Halperin, Nachshon, and Carmon (1973). These authors asked listeners to label dichotic tone triads, each tone in a triad being either high $(\mathrm{H})$ or low $(\mathrm{L})$ in pitch (long or short in duration for a second condition); they found that a left-ear advantage for homogeneous triads (e.g., HHH or LLL) shifted increasingly to a right-ear advantage as the number of "transitions" increased from one (e.g., HLL, HHL, etc.) to two (e.g., HLH, LHL). Halperin et al. (1973) concluded that "perception of temporal patterns might be one of the underlying mechanisms in speech perception" (p. 46).

Perhaps Tallal and Newcombe (1978) were misled by the unhappy coincidence of the use of the word "transition" to describe both Halperin et al.'s temporal patterns and the spectral sweeps at the onset of stop-vowel syllables. In any event, even if patterns of discrete-pitch change, as in Tallal's or Halperin et al.'s tone-sequencing tasks, can properly be described as temporal, the continuous sweeps of stop-vowel transitions certainly can not be. To see this, we must take a brief detour into the problem of coarticulation.

Coarticulation refers to the overlapping articulation of two or more neighboring segments (consonants or vowels). A prototypical example is a consonant-vowel-consonant (CVC) syllable, such as the word bag (Liberman, 1970; see also Liberman, Cooper, Shankweiler, \& Studdert-Kennedy, 1967), in which so-called "perseveratory" effects of the initial /b/ and "anticipatory" effects of the final $/ g /$ are distributed across the entire vowel. As a result, every portion of the syllable, both articulatorily and acoustically, carries information simultaneously about more than one segment. The syllable is then a unit of spatiotemporal interaction among articulators, and its integral acoustic form conveys information about successive segments in parallel rather than in series. Thus, the rapidly changing resonances of the vocal tract (formant transitions) at the onset of a stopvowel syllable convey information about both consonant and vowel. Moreover, the transitions of synthetic $/ \mathrm{ba} /$ and $/ \mathrm{da} /$, used by Tallal, have identical temporal properties (duration, rate of frequency change); they differ in the loci and directions of their frequency trajectories. The contrast is therefore spectral, not temporal, and it is spectral, not temporal, sensitivity that perception of the contrast requires. (For textbook discussions of coarticulation and the problems it raises for speech perception, see Borden, Harris, \& Raphael, 1994, chap. 5, and Pickett, 1980, chap. 10.)

Here, then, is the start of the conceptual confusion in the "temporal processing" hypothesis. The trouble begins when Tallal and Newcombe (1978) completely reverse, without evidence or explanation, the conclusions of Tallal and Piercy $(1973,1974,1975)$. They do this (1) by equating "temporal perception" with rapid perception, and (2) by attributing the dysphasic children's difficulties to the transitional character rather than the brevity of the formant transitions. They then compound the muddle by adopting such phrases as "rapidly changing acoustic ... 
information" (Tallal \& Newcombe, 1978, p. 19) to describe both the temporal patterns of discrete tone sequences and the continuous spectral sweeps of formant transitions.

We turn now to see how Farmer and Klein, seemingly unaware of contradictions in the hypothesis they have undertaken to evaluate, handle the three issues that emerged from the work of Tallal and Piercy.

\section{DEFICITS IN DISCRIMINATIVE CAPACITY, NOT IN TOJ}

Farmer and Klein divide "sequential processing" into three components: stimulus identification (Table 1), gap detection (Table 2), and TOJ (Table 3). Of these, only the second is necessarily temporal. Stimulus identification is, of course, prerequisite to TOJ, but would itself be temporal only if the defining property of the event to be identified was temporal. The stimulus-identification studies selected for Table 1 reveal how confusion between tone sequences and formant transitions has ramified through the target paper: Farmer and Klein omit all studies of synthetic stop-consonant continua, because "such phonemes are not regarded as single stimuli, but as a series of rapidly changing acoustic events" (p. 465). They do refer to some of these studies at a later point (p. 482), but by omitting them from Table 1, Farmer and Klein lose an opportunity to focus attention on the single most important body of work concerning speech-perception deficits in the readingimpaired, namely, some half a dozen studies reporting that identification along synthetic continua is less consistent among poor or dyslexic readers than among controls (e.g., De Weirdt, 1988; Godfrey, Syrdal-Lasky, Millay, \& Knox, 1981; Pallay, 1986; Reed, 1989; Steffens, Eilers, GrossGlen, \& Jallad, 1992; Werker \& Tees, 1987). In several of these studies where discrimination was also tested, impaired readers performed significantly worse than normal controls between categories but not within, indicating that they could not easily exploit the phonological contrast that normally enhances discrimination across a phoneme boundary. Thus, their difficulties were in identifying and discriminating phonologically contrastive, but phonetically similar, synthetic syllables.

Such results suggest that phonological categories may be less sharply defined in reading-impaired than in normal children (cf. Reed, 1989, p. 288). Their omission from Table 1 impedes the reader's recognition of deficits in phonetic discriminative capacity that predict precisely the difficulties on $/ \mathrm{ba} /-/ \mathrm{da} /$ TOJ observed by Reed (1989) in reading-impaired children (Table 3), and that support the nontemporal account of TOJ deficits favored by Tallal herself (e.g., 1980, Table 3). One further study, not mentioned by Farmer and Klein, also supports this account. Watson and Miller (1993) found that, although readingdisabled undergraduates made significantly more errors than normal readers on a test of nonspeech tone TOJs, three other tests, which (unlike tone TOJ) had clear face va- lidity as measures of auditory temporal perception, did not distinguish between the groups. Table 3 , then, lists apparent TOJ deficits among the reading-impaired as reported for nonspeech tones by two studies (Reed, 1989; Tallal, 1980), for nonspeech auditory clicks by one study (Kinsbourne, Rufo, Gamzu, Palmer, \& Berliner, 1991), and for speech sounds $(/ \mathrm{ba} /-/ \mathrm{da} /)$ by one (Reed, 1989). All are consistent with the view that the deficits are in discrimination/ identification, not in temporal perception itself.

For the rest, only 1 study (McCroskey \& Kidder, 1980 , listed in Table 2) of the 20 studies ( 5 auditory, 15 visual) listed in Tables 1,2, and 3 reports an unambiguous deficit in auditory temporal perception in reading-impaired subjects, and it is far from clear how this deficit relates to speech perception. Yet Farmer and Klein summarily conclude: "In short, there is compelling evidence in groups of dyslexics for a deficit in TOJs in the auditory domain" (p. 469). This statement grossly misrepresents the facts.

\section{PERCEIVING FORMANT TRANSITIONS}

As we have seen, no evidence beyond the assertions of Tallal and Newcombe (1978) supports the claim that some impaired children have difficulty with the "rapid acoustic changes" of formant transitions. Nonetheless, the target paper offers a speculative account of how such a difficulty might arise. Farmer and Klein apparently accept without question that transitions are equivalent to tone sequences, and that their perception is a matter of temporal order judgment. Thus, referring to the patterns along a synthetic stop consonant continuum, they write, as already quoted: "Such phonemes are not regarded as single stimuli, but as a series of rapidly changing acoustic events (the spectral changes of the formant transitions)" (p. 465), and later, to explain how a deficit in sequencing ability might affect speech perception: "The stop consonants involve spectral changes in the time frame of tens of milliseconds, and any impairment in the ability to process the order of these changes would result in impaired discrimination of the sounds" (p. 467; our italics). These statements include at least three points of misunderstanding. First, a CV formant transition is not "a series of rapidly changing acoustic events," but an integral spectral sweep reflecting the continuously changing resonances of the vocal tract, as a speaker moves from a point of closure into the following vowel. Second, as many experiments have shown (e.g., Mann \& Liberman, 1983; Mattingly, Liberman, Syrdal, \& Halwes, 1971), a brief formant transition removed from the speech signal is heard as a rapid, integral glissando, or "chirp," of which the parts or "spectral changes" cannot be perceptually "individuated," as Farmer and Klein's own account of TOJ would require. Third, even if a temporal order error in perception of a transition were possible, the resulting percept, since the transition begins with consonant release and ends in the vowel nucleus, would presumably reverse these segments, yielding /ab/ for $/ \mathrm{ba} /$ or $/ a d /$ for $/ \mathrm{da} /$. Studies of speech errors never report such 
within-syllable metatheses in either production or perception. In short, Farmer and Klein's notion that a deficit in TOJ capacity would cause a failure to perceive formant transitions is untenable.

\section{THE RELATION BETWEEN DEFICITS IN SPEECH AND NON-SPEECH AUDITORY PERCEPTION}

\section{Hemispheric Specialization}

The claim that phonological deficits are auditory in origin (Farmer \& Klein, 1995, pp. 480-483) would be encouraged by evidence that the well-established specialization of the left hemisphere for speech perception is grounded in a prior specialization for aspects of auditory perception essential to speech. We now briefly review claims for such evidence from dichotic and aphasic studies.

Dichotic studies. A key paper, cited by Farmer and Klein, comes from Schwartz and Tallal (1980). In this paper, a right-ear advantage (REA) for synthetic stop consonantvowel syllables was significantly reduced when the initial transitions were lengthened from 40 to $80 \mathrm{msec}$. Farmer and Klein follow the authors in interpreting this result as evidence for left-hemisphere dominance in processing rapidly changing acoustic events. However, two conditions are necessary for an ear advantage: (1) hemispheric specialization, and (2) fuller access to the specialized hemisphere from the contralateral than from the ipsilateral ear (Shankweiler \& Studdert-Kennedy, 1967; StuddertKennedy \& Shankweiler, 1970, 1981). Variations in the magnitude of the REA within or between different classes of speech sound are therefore ambiguous: Are they due to differences in degree of hemispheric specialization (presumably, a more or less stable property of brain structure and function) or to differences in contralateral access (at least in part, a variable aspect of perceptual function)? Many studies have shown that the size and even direction of an ear advantage can be manipulated experimentally (see Studdert-Kennedy \& Shankweiler, 1981, for references). The most straightforward interpretation of Schwartz and Tallal (1980) therefore is not that they reduced the degree of left-hemisphere engagement by increasing the duration of the onset transitions, but that they simply raised the salience of the ipsilateral signal, and so reduced the ear advantage. The latter is the more plausible interpretation, because we do not then have to suppose that in normal listening the left hemisphere is more engaged by some portions of the speech signal than by others, or that its degree of engagement varies with the duration or intensity of the signal.

Nonspeech dichotic studies purporting to show specialization of the left hemisphere for temporal processing are no less ambiguous, often because we cannot rule out covert verbal mediation (e.g., Halperin et al., 1973, cited above). The only dichotic study cited by Farmer and Klein as "evidence of an REA for the processing of temporally complex nonspeech sounds" (p. 481) was Divenyi and Efron's (1979), which actually used 100-msec steady-state pure tones; they were judged for pitch and yielded a LEA in 5 out of 6 subjects.
Finally, the notion that specialization of the left hemisphere rests on a capacity for processing a particular type of physical stimulus is belied by dichotic studies in which identical stimuli give rise to different ear advantages as a function of their status in the listeners' languages (e.g., Avery \& Best, 1994; van Lancker \& Fromkin, 1973).

Aphasic studies. In the study cited above, Tallal and Newcombe (1978) undertook to determine whether TOJ deficits of the kind observed in dysphasic children by Tallal and Piercy $(1973,1974,1975)$ were associated with left- or right-hemisphere lesions in adults. They found that (1) left-hemisphere patients were significantly worse than right-hemisphere patients or normal controls on rapid TOJ for tones and synthetic $/ \mathrm{ba} /-/ \mathrm{da} /$, but not on long, steadystate vowels; (2) while only 3 out of 10 left-hemisphere patients could identify $/ \mathrm{ba} /-/ \mathrm{da} /$ with 40 -msec transitions, 7 out of 10 could do so when the syllables had 80 -msec transitions; (3) for left-hemisphere patients rank order correlation between errors on tone TOJ and on a test of language comprehension was highly significant. Tallal and Newcombe (1978) thereforc concluded, as already quoted, that "the left hemisphere must play a primary role in the analysis of specific rapidly changing acoustic cues, verbal and nonverbal, and ... such analysis is critically involved in both the development and maintainance [sic] of language" (p. 19).

Yet, as we have seen, the supposed deficit "in the analysis of specific rapidly changing acoustic cues, verbal and nonverbal" is based on unwarranted equation of tone sequences with formant transitions. Moreover, attempts to replicate the effect of lengthened transitions with adult aphasics have not been successful (Blumstein, Tartter, Nigro, \& Statlender, 1984; Riedel \& Studdert-Kennedy, 1985; cf. note 1). Finally, as often in aphasic studies, interpretation of the correlation is unsure: was the deficit underlying difficulties with TOJ a cause or a consequence of the language deficit? In well-known related studies, Efron (1963) and Swisher and Hirsh (1972) observed nonspeech TOJ deficits in aphasics, but explicitly rejected causal interpretations.

Stronger than these arguments, however, are the results of an experimental test of Tallal and Newcombe's (1978) claims by Aram and Ekelman (1988). They compared 20 left- and 12 right-brain-lesioned children on Tallal's tonediscrimination and TOJ tests. The test materials were prepared in Tallal's laboratory, and testers were trained in the procedures of test administration by Tallal and her staff. Yet Aram and Ekelman found no differences between the lesioned children and normal controls or between the leftand right-lesioned children. Nor did they find any relation between the performance of the left-lesioned children on the tone tasks and various language tasks. They concluded that "the higher level language deficits seen in left brain lesioned children cannot be attributed to difficulty in more preliminary analyses of the acoustic stimuli" (p. 935).

Farmer and Klein dismiss this work in a footnote on the grounds that the children represented "quite a different population from the developmentally language-impaired children studied by Tallal" (note 5, p. 492). So, of course, 
did the adult aphasics of Tallal and Newcombe (1978); yet those authors did not hesitate to generalize their findings to the children of Tallal and Piercy $(1973,1974,1975)$. Moreover, if the left hemisphere does indeed "play a critical role" in temporal analysis, if such analysis is indeed "critical to the development and maintenance of language," and if Tallal's tests do indeed measure this lefthemisphere capacity, we would surely expect that leftlesioned children with serious language deficits would have difficulty with those tests. Farmer and Klein's disclaimer therefore strikes us as less than compelling.

\section{A Control Study}

Conspicuously absent from Tallal's work, in the nearly 20 years that have elapsed since Tallal and Newcombe (1978) first made the claim, is any attempt to test the auditory basis of the supposed deficit in the perception of formant transitions by means of an appropriate nonspeech control. The required control has come from Mody (1993; Mody, Studdert-Kennedy, \& Brady, 1995). Her readingimpaired subjects were 20 second-grade children, reading at least 5 months below grade level, and selected for their significant number of errors on Tallal's / ba/-/da/TOJ task. In Experiment 1a, the subjects were tested on discrimination and TOJ of synthetic $/ \mathrm{ba} /-/ \mathrm{da} /$ at short ISIs; errors increased monotonically as ISI decreased on both discrimination and TOJ, with no significant difference between tasks. In Experiment $1 \mathrm{~b}$, the same procedure was followed, with syllable pairs $/ \mathrm{ba} /-/ \mathrm{sa} /$ for one half of the group and $/ \mathrm{da} / / / \int_{\mathrm{a}} /$ for the other half; they made almost no errors on either task. Thus, despite their difficulties with /ba/-/da/, they performed perfectly under time pressure when they could clearly identify the syllables to be ordered, a result consistent with Tallal's (1980) view of TOJ. Evidently $/ \mathrm{ba} /-/ \mathrm{da} /$ were difficult to discriminate and identify in rapid succession because they are very similar.

Is the similarity of $/ \mathrm{ba} /$ and $/ \mathrm{da} /$ auditory or phonetic? Experiment 2 was designed to answer this question. Nonspeech control stimuli were synthesized. They consisted of two sine waves with durations and frequency trajectories identical to those of the center frequencies of $F 2$ and F3 that carried the $/ \mathrm{ba} /-/$ da/ contrast in Experiment 1 . Perceptually, the stimuli did not resemble their speech models. After preliminary identification training, subjects were tested for discrimination at the short ISIs of Experiment 1 . Contrary to the results for $/ \mathrm{ba} /-/ \mathrm{da} /$, performance was completely unaffected by decreases in ISI.

The combined results of the two experiments demonstrate that the reading-impaired children's difficulties with $\mathrm{ba} /-/ \mathrm{da} /$ were due neither to a general deficit in rate of auditory processing nor to difficulties in processing brief patterns of rapidly changing acoustic information, but rather to difficulties in identifying similar syllables, presented in rapid succession. Since the nonspeech control patterns of Experiment 2 were as acoustically similar as the $/ \mathrm{ba} /$ and $/ \mathrm{da} /$ of Experiment 1 , it would seem that $/ \mathrm{ba} /$ and $/ \mathrm{da} /$ are difficult to identify at rapid presentation rates because, although phonologically contrastive, they are phonetically similar; like speech sounds at the poles of a synthetic continuum which, as mentioned above, impaired readers often cannot readily identify, they differ on a single phonetic feature.

We cannot evade the results of this study (as Farmer and Klein hoped to evade the results of Aram \& Ekelman, 1988 ) by arguing that the sampled population somehow differed from the population sampled in other studies. To be sure, the poor readers of this study were not dyslexic, or even as impaired as the poor readers of Tallal (1980). They did display, however, precisely the difficulties with $/ \mathrm{ba} /-/ \mathrm{da} /$ TOJ that Reed (1989) observed in some readingimpaired children and that Tallal (1980) proposed as symptomatic of a phonological disorder in such children. Yet, the source of those difficulties in the children of Mody (1993) was definitely not an inability to perceive brief formant transitions. Unless we are willing to suppose that perceptual difficulties in readers who read half a year behind grade level (Mody, 1993) have different causes than do identical difficulties attributed to readers who read a full year behind grade level (Tallal, 1980), we have to concede that difficulties in identifying $/ \mathrm{ba} /-/ \mathrm{da} /$ at rapid rates of presentation have nothing to do with the transitional properties of their onsets (ironically, the very conclusion of Tallal and Piercy, 1975!) and are phonetic, not auditory, in origin.

\section{CONCLUSION}

The hypothesis that impaired readers' phonological deficits stem from a left-hemisphere deficit in auditory temporal perception rests on conceptual confusion between temporal perception and rapid perception, and on misinterpretation of data from dichotic experiments and aphasic studies. The difficulties of some impaired readers with rapid temporal order judgments in speech and/or nonspeech seem to reflect independent deficits in discriminative capacity of unknown origin, not a general deficit in either "temporal processing" or rate of auditory perception. We conclude that, on the available evidence, the phonological deficit of impaired readers cannot be traced to any co-occurring nonspeech deficits so far observed and is phonetic in origin, but that its full nature, origin, and extent remain to be determined.

\section{REFERENCES}

Aram, D. M., \& Ekelman, B. L. (1988). Auditory temporal perception of children with left or right brain lesions. Neuropsychologia, 26,931935.

AvERY, R. A., \& Best, C. T. (1994). Hemispheric differences in the perception of Zulu click consonants. Journal of the Acoustical Society of America, 96. 3230 .

Blumstein, S., Tartter, V., Nigro, G., \& Statlender, S. (1984). Acoustic cues for the perception of place of articulation in aphasia. Brain \& Language, 22, 128-149.

Borden, G., Harris, K. S., \& Raphael, L. J. (1994). Speech science primer (3rd ed.). Baltimore: Williams \& Wilkins. 
DE WEIRDT, W. (1988). Speech perception and frequency discrimination in good and poor readers. Applied Psycholinguistics, 16, 163-183. DivenYi, P. L., \& EFRON, R. (1979). Spectral versus temporal features in dichotic listening. Brain \& Language, 7, 375-386.

EFron, R. (1963). Temporal perception, aphasia and déjà vu. Brain, 86, 403-424.

Farmer, M. E., \& Klein, R. M. (1995). The evidence for a temporal processing deficit linked to dyslexia: A review. Psychonomic Bulletin \& Review, 2, 460-493.

Godfrey, J. J., Syrdal-Lasky, A. K., Millay, K. K., \& Knox, C. M (1981). Performance of dyslexic children on speech perception tests. Journal of Experimental Child Psychology, 32, 401-424.

Halperin, G., NaChshon, I., \& Carmon, A. (1973). Shift of ear superiority in dichotic listening to temporally patterned nonverbal stimuli Journal of the Acoustical Society of America, 53, 46-50.

Kinsbourne, M., Rufo, D. T., Gamzu, E., Palmer, R. L., \& Berliner, A. K. (1991). Neuropsychological deficits in adults with dyslexia. Developmental Medicine \& Child Neurology, 33, 763-775.

Liberman, A. M. (1970). The grammars of speech and language. Cognitive Psychology, 1, 301-323.

Liberman, A. M., Cooper, F. S., Shankweiler, D., \& StuddertKennedy, M. (1967). Perception of the speech code. Psychological Review, 74, 431-461.

ManN, V. A., \& Liberman, A. M. (1983). Some differences between phonetic and auditory modes of perception. Cognition, 14, 211-235.

Mattingly, I. G., Liberman, A. M., Syrdal, A. K., \& Halwes, T. (1971). Discrimination in speech and non-speech modes. Cognitive Psychology, 2, 419-430.

MCCroskey, R. L., \& Kidder, H. C. (1980), Auditory fusion among learning disabled, reading disabled, and normal children. Journal of Learning Disabilities, 13, 18-25.

MoDy, M. (1993). Bases of reading impairment in speech perception: $A$ deficit in rate of auditory processing or in phonological coding? Unpublished doctoral dissertation, City University of New York.

Mody, M., StudDerT-KenNedy, M., \& BRAdy, S. (1995). Speech perception deficits in poor readers: Auditory processing or phonological coding? Manuscript submitted for publication.

Pallay, S. L. (1986). Speech perception in dyslexic children. Unpublished doctoral dissertation, City University of New York.

PicketT, J. M. (1980). The sounds of speech communication. Baltimore: University Park Press.

REED, M. A. (1989). Speech perception and the discrimination of brief auditory cues in reading disabled children. Journal of Experimental Child Psychology, 48, 270-292.

Riedel, K., \& StudderT-Kennedy, M. (1985). Extending formant transitions may not improve aphasic's perception of stop consonant place of articulation. Brain \& Language, 24, 223-232.

SChWartz, J., \& TALLAL, P. (1980, March 21). Rate of acoustic change may underlie hemispheric specialization for speech perception. Science, 207, 1380-1381.

ShanKWEILER, D. P., \& STUDDERT-KenNEDY, M. (1967). Identification of consonants and vowels presented to left and right ears. Quarterly Journal of Experimental Psychology, 19, 59-63.

Steffens, M. L., Eilers, R., Gross-Glen, K., \& Jallad, B. (1992). Speech perception deficits in adult subjects with familial dyslexia. Journal of Speech \& Hearing Research, 35, 192-200.
Studdert-Kennedy, M., \& Shankweiler, D. P. (1970). Hemispheric specialization for speech perception. Journal of the Acoustical Society of America, 48, 579-594.

Studdert-Kennedy, M., \& Shankweiler, D. [P.] (1981, February 27). Hemispheric specialization for language processes. Science, $\mathbf{2 1 1}$, 960-961.

Swisher, L., \& HirSH, I. J. (1972). Brain damage and the ordering of two temporally successive stimuli. Neuropsychologia, 10, 137-152.

TALLAL, P. (1980). Auditory temporal perception, phonics and reading disabilities in children. Brain \& Language, 9, 182-198.

Tallal, P. (1984). Temporal or phonetic processing deficit in dyslexia? That is the question. Applied Psycholinguistics, 5, 167-169.

Tallal, P., Miller, S., \& Fitch, R. H. (1993). Neurobiological basis of speech: A case for the preeminence of temporal processing. In P. Tallal, A. M. Galaburda, R. R. Llinas, \& C. von Euler (Eds.), Temporal information processing in the nervous system (Annals of the New York Academy of Sciences, Vol. 82, pp. 27-47). New York: New York Academy of Sciences.

TALlAL, P., \& NEWCOMBE, F. (1978). Impairment of auditory perception and language comprehension in dysphasia. Brain \& Language, 5, 13-24.

TAllal, P., \& PIERCY, M. (1973). Developmental aphasia: Impaired rate of nonverbal processing as a function of sensory modality. Neuropsychologia, 11, 389-398.

Tallal, P., \& Piercy, M. (1974). Developmental aphasia: Rate of auditory processing and selective impairment of consonant perception. Neuropsychologia, 12, 83-93.

Tallal, P., \& PierCy, M. (1975). Developmental aphasia: The perception of brief vowels and extended stop consonants. Neuropsychologia, 13, 69-74.

Tallal, P., Sainburg, R. L., \& Jernigan, T. (1991). The neuropathology of developmental dysphasia: Behavioral, morphological and physiological evidence for a pervasive temporal processing disorder. Reading \& Writing: An Interdisciplinary Journal, 3, 363-377.

VAN LANCKER, D., \& Fromkin, V. A. (1973). Hemispheric specialization for pitch and "tone": Evidence from Thai. Journal of Phonetics, $1,101-109$

WATSON, B. U., \& Miller, T. K. (1993). Auditory perception, phonological processing and reading ability/disability. Journal of Speech \& Hearing Research, 36, 850-863

WerkER, J., \& TEES, R. (1987). Speech perception in severely disabled and average reading children. Canadian Journal of Psychology, 41, 48-61.

\section{NOTE}

1. Lengthening formant transitions from 30 to $80 \mathrm{msec}$ shifts the phonetic quality of the phonological contrast from stop to glide (Borden, Harris, \& Raphael, 1994, chap. 6; Pickett, 1980, chap. 6; cf. Riedel \& Studdert-Kennedy, 1985), and is therefore not purely auditory in its effect. No one, so far as we know, has replicated the findings of Tallal and Piercy (1975) with specifically reading-impaired children.

(Manuscript received April 25, 1995; revision accepted for publication May 31, 1995.) 